\title{
LOFT 2010 Conference on Logic and the Foundations of Game and Decision Theory, University of Toulouse, France, 5-7 July 2010
}

\section{Aims of the conference}

This is the ninth in a series of conferences on the applications of logical methods to foundational issues in the theory of individual and interactive decision-making. Preference is given to papers which bring together the work and problems of several fields, such as game and decision theory, logic, computer science and artificial intelligence, philosophy, cognitive psychology, mathematics and mind sciences.

Topics of particular relevance are:

(Modal) logics for games and protocols: epistemic and deontic logic, multi-agent logic, temporal logic, dynamic logic, probabilistic and multivalued logic, logic of belief revision.

Foundations of game and decision theory: epistemic foundations of solution concepts, information processing and communication in games, belief formation and revision in games.

Learning and information-processing models: economic aspects of information processing, learning in game-theoretic contexts, inductive learning and inductive decision making.

Bounded rationality approaches to game and decision theory.

For previous conferences and related publications see

http://www.econ.ucdavis.edu/faculty/bonanno/loft.html.

\section{Submission information}

Extended abstract of 5-10 pages in PDF format should be submitted at: http://www.easychair.org/conferences/?conf=loft2010.

Deadline for submission: 15 March 2010, Notification: 18 April 2010

A selection of papers presented at LOFT9 will be published in a special issue of the International Journal of Game Theory.

Program Chairs:

Giacomo Bonanno, University of California Davis, USA

Andreas Herzig, University of Toulouse, France

Wiebe van der Hoek, University of Liverpool, UK

Jerome Lang, University Paris-Dauphine and University of Toulouse, France 